ESJ Humanities

\title{
Le triptyque uti possidetis, titre et effectivités dans la délimitation des frontières par le juge international en Afrique
}

\author{
Pauline Solange Mevah Bikongo
}

Docteur/Ph.D en droit public à l'Université de Yaoundé II, Cameroun

Doi:10.19044/esj.2021.v17n6p29

Submitted: 05 May 2020

Accepted: 25 January 2021

Published: 28 February 2021
Copyright 2021 Author(s)

Under Creative Commons BY-NC-ND

4.0 OPEN ACCESS

Cite As:

Mevah Bikongo P.S. (2021). Le triptyque uti possidetis, titre et effectivités dans la délimitation des frontières par le juge international en Afrique. European Scientific Journal, ESJ, 17(6), 29. https://doi.org/10.19044/esj.2021.v17n6p29

\section{Résumé}

Si l'indépendance a été un défi fondamental pour l'Afrique hier et qu'aujourd'hui le développement économique ou l'émergence sonne comme un impératif catégorique, il existe un autre défi et non des moindres, celui de la pacification de l'Afrique qui, depuis les indépendances, brille justement par des foyers interminables de crises. Mais il est de notoriété publique que l'une des causes de ces crises est la revendication des territoires par les Etats, car les frontières ont, pour la plupart, été mal définies au moment du partage du gâteau orchestré par la non moins célèbre Conférence de Berlin. Pour sauver les meubles, et en conformité avec les principes des Nations Unies auxquels tous les Etats africains avaient adhéré, notamment le principe de non recours à la force dans les relations internationales et le principe de règlement pacifique des différends internationaux, les Etats africains ont eu recours au juge international pour régler leurs différends frontaliers. Dans son office, le juge, qu'il soit de la CIJ ou d'un tribunal arbitral, a très souvent fondé sa décision sur l'un des trois éléments majeurs que sont le titre juridique, les effectivités et l'uti possidetis. Si les deux premiers éléments sont des classiques sur lesquels le juge international a toujours fondé ses décisions en matière frontalière, l'uti possidetis en tant que principe général de droit, consacré aussi bien par la Charte de l'OUA que celle UA, semble être l'élément de référence devant orienter la décision du juge. Cependant, à bien scruter la jurisprudence internationale, il ressort que le juge fait usage de ces 
trois concepts dans la délimitation des frontières en Afrique, au point qu'ils paraissent dans une imbrication trinitaire.

Mots-clés : Titre juridique, effectivités, uti possidetis/intangibilité des frontières, frontière, délimitation

\title{
The Triptych Uti Possidetis, Title and Effectivités in the Delimitation of Borders by the International Judge in Africa
}

\author{
Pauline Solange Mevah Bikongo \\ Docteur/Ph.D en droit public à l'Université de Yaoundé II, Cameroun
}

\begin{abstract}
If independence was a fundamental challenge for Africa yesterday and today economic development or emergence sounds like a categorical imperative, there is another challenge and not least, that of the pacification of Africa which, since independence, has been shining through endless hotbeds of crises. But it is common knowledge that one of the causes of these crises is the claim of the territories by the States, because the borders were for the most part badly defined at the time of the sharing of the cake orchestrated by the no less famous Berlin Conference. To save the furniture, and in accordance with the principles of the United Nations to which all the African States had adhered, in particular the principle of not recourse to force in the international relations and the principle of peaceful resolution of the international disputes, the African States had recourse to the international judge to settle their border disputes. In his office, the judge, whether from the ICJ or from an arbitral tribunal, very often based his decision on one of the three major elements: legal title, effectivités or uti possidetis. If the first two elements are classics on which the international judge has always based his decisions in border matters, the uti possidetis has, by virtue of its status as a treaty principle enshrined in the Charter of the OAU / AU, seemed to be the reference element to guide the judge's decision. However, if you look closely at international jurisprudence, it turns out that the judge makes alternating use of these three principles in Africa, to the point that they appear in a Trinitarian overlap.
\end{abstract}

Keywords: Legal title, effectivités, uti possidetis / intangibility of borders, border, delimitation

\section{Introduction}

Les frontières étant des lignes qui déterminent là où commencent et où finissent les territoires relevant respectivement d'Etats voisins 
(BASDEVANT, 1960), elles sont liées à l'existence des Etats en ceci que ce sont elles qui délimitent le territoire occupé par l'Etat et circonscrivent le lieu d'exercice de sa souveraineté. Ainsi pour délimiter une frontière, les Etats limitrophes peuvent procéder par une entente en signant un accord de délimitation (délimitation négociée), ou alors se présenter devant un juge pour lui demander de tracer leur frontière (délimitation décidée), (KAMGA, 2006).

Généralement les Etats envisagent la deuxième option lorsqu'ils n'arrivent pas à s'entendre sur le lieu de passage de la ligne frontière qui les sépare. Etant donné que cette mésentente les amène souvent à entrer en conflit, pour y trouver une solution, ils optent pour la saisine du juge. En effet, le juge international joue un rôle important dans le processus de délimitation des frontières en Afrique. Il officie lorsque les Etats font recours à lui pour trancher le différend frontalier qui les oppose.

Il faut toutefois noter qu'en ce qui concerne le cas du continent africain, ces différends frontaliers trouvent leur origine dans le partage de l'Afrique effectué en Allemagne lors de la Conférence de Berlin tenue du 15 novembre 1884 du 22 février 1885. C'est lors de cette conférence que les puissances coloniales, pour mettre fin aux rivalités qui les opposaient sur le continent, ont procédé au partage de celui-ci (LOULICHKI, 2018), en l'absence des principaux concernés, à savoir les peuples africains. Ainsi, les frontières africaines ont été tracées sur la base des cartes dont les puissances coloniales disposaient, en ignorant la réalité sur le terrain. Comme l'indique à ce propos Lord Salisbury, l'un des acteurs majeurs du partage : Nous avons entrepris de tracer sur les cartes des régions où l'homme blanc n'avait jamais mis pied. Nous nous sommes distribué des montagnes, des rivières et des lacs à peine gênés par cette petite difficulté que nous ne savions jamais exactement où se trouvaient ces montagnes, ces rivières ou ces lacs (PERSON, 1972). Cet imbroglio a perduré pendant tout le temps de la colonisation. De telle manière qu'une fois indépendants, les Etats africains, successeurs des empires coloniaux, ont également hérité de cette relativité du tracé des frontières coloniales. Cet état des choses a conduit les nouveaux Etats africains à des conflits, chacun revendiquant la connaissance du passage de la ligne frontière. La cacophonie qui en a suivi aurait pu ouvrir la brèche à des conflits armés qui auraient constitué des véritables menaces pour la paix, n'eue été le reflexe développé par les gouvernements africains de saisir le juge international pour rétablir l'ordre.

Pour ce faire, le juge international, pour assurer un tracé définitif des frontières légitimes a très souvent eu recours à trois éléments déterminants : le titre juridique, les effectivités et le principe consacré par l'OUA (l'Organisation de l'Unité Africaine) d'intangibilité des frontières héritées de la colonisation, encore connu sous l'appellation uti possidetis. Avant de s'y pencher dans les développements qui vont suivre, il faut relever à titre de 
précision terminologique sommaire que le titre juridique renvoie à la preuve documentaire qui peut être un traité international, un accord de délimitation ou tout autre texte authentique qui atteste de la délimitation d'une frontière. Les effectivités quand à elles se réfèrent à des comportements qui témoignent d'une certaine maîtrise du territoire dont il est question; il s'agit des comportements ou des éléments de preuve sur l'administration effective du territoire querellé par l'une des parties. Cela peut équivaloir en droit foncier au principe de mise en valeur d'un terrain. L'uti possidetis quant à lui est un principe d'origine sud américaine, mais, consacré par l'OUA dans sa Charte Constitutive, consistant en un engagement des Etats africains de ne jamais remettre en cause les frontières coloniales ; c'est ce qu'on a appelé le principe d'intangibilité des frontières coloniales.

Cependant, une certaine polémique est née sur la signification de 'l'intangibilité des frontières" qui signifie en principe 'immutabilité", ou "immuabilité"' ce qui ne pourrait donc pas, de l'avis de certains auteurs, être assimilé à ' 'l'uti possidetis". Dans la mesure où ce serait un "non-sens », (BARDONNET, 1979). Car, l'uti possidetis juris ne suppose pas que les frontières restent figées vu qu'elles sont toujours susceptibles de modification par voie d'accord, (ABLINE, 2006). Ces auteurs estiment que, l'uti possidetis juris est donc abusivement assimilé à l'intangibilité des frontières. Pour Klein "'l' intangibilité des frontières" ici est une expression trompeuse car elle renvoie à une idée d'immutabilité que l'uti possidetis ne tend pas à assurer, (KLEIN, 1999). Fort heureusement, la CIJ dans l'Affaire du différend frontalier Benin/Niger du 12 juillet 2005, reconnait l'identité de ces deux concepts lorsqu'elle déclare que : Ainsi que la chambre l'a rappelé (voir paragraphe 23 ci-dessus), les parties lui ont expressément demandé de s'acquitter de sa tâche en appliquant en particulier, le principe de la succession des Etats aux frontières héritées de la colonisation, à savoir le principe de l'intangibilité des dites frontières, ou encore principe de l'uti possidetis juris ».

Ceci étant la question principale qui motive notre réflexion est celle de savoir quelle est l'importance que le juge international accorde à chacun des éléments du triptyque titre, effectivités et uti possidetis dans le règlement des différends frontaliers en Afrique. La réponse à cette question éminemment délicate, en ce sens qu'elle nous impose une lucidité digne des positivistesdogmatiques, a transité par une analyse documentaire importante.

Cependant, sans prétendre à l'exhaustivité dans nos recherches, la lecture de l'abondante jurisprudence de la CIJ (Cour internationale de Justice) et des tribunaux internationaux en la matière nous a permis de constater que le juge international accorde à ces trois éléments déterminants une importance alternante, en fonction de leur pertinence. Cependant, bien qu'il semble faire la part belle à l'uti possidetis qui est un principe fondamental consacré par la 
Charte de l'OUA devenue UA (I), pour tracer la frontière, il fonde toujours ses décisions sur les éléments classiques que sont le titre et les effectivités (II).

\section{Uti Possidetis ou L'intangibilite des Frontieres Coloniales: Base Fondamentale Determinant L'orientation de la Decision du Juge}

En matière de délimitation des frontières post coloniales, l'uti possidetis a été envisagé comme la norme à suivre en la matière en Afrique. Par ce principe, les Etats nouvellement indépendants s'engagent à conserver comme frontières, les limites qui étaient celles des territoires dont ils sont issus. C'est ainsi qu'il trouve ses origines en Amérique latine (A) avant d'être appliqué en Afrique (B).

\section{A. Les Origines du Principe}

L'uti possidetis trouve son origine dans le droit romain dont la formule est uti possidetis, ita possedetis qui signifie "'continue de posséder ce que tu possédais'. Il visait à attribuer la possession provisoire du bien en cause à la partie qui le possédait à ce moment-là. Ce principe refait surface en Amérique latine (1), mais sous une autre forme avec des rôles similaires (2).

\section{L'origine latine du principe}

Ce principe est mis en place dans le processus de séparation des Républiques d'Amérique du Sud en 1810 suivi de la désunion de l'Amérique centrale en 1821 d'avec les puissances coloniales européennes, à savoir l'Espagne et le Portugal. Ces Etats, voulant s'émanciper des colons, adoptèrent le principe de l'uti possidetis comme instrument de délimitation. En effet, c'est lors du congrès d'Angostura en 1819 que les jeunes Etats décidèrent que leurs frontières coïncideraient avec celles de l'administration coloniale telles qu'elles existaient en 1810 (MEHDI, 1998). Ce principe fut ainsi consacré dans plusieurs textes juridiques à l'instar des constitutions et des conventions. On peut citer ainsi : l'article 2 de la loi fondamentale du 17 décembre 1819 régissant la Colombie, l'Equateur et le Venezuela, l'article 2 de la loi fondamentale de la Nouvelle-grenade du 30 novembre 1831, l'article 3 de la constitution colombienne du 4 août 1886, l'article 7 du protocole de Lima de 1847 qui énonçait que «Les Républiques de la confédération reconnaissent l'uti possidetis comme principe fondé en droit pour la détermination de leurs frontières respectives ». L'article 2 paragraphe $3 \mathrm{du}$ traité de 1894 relatif à la frontière entre le Nicaragua et le Honduras dispose que "Chaque République est maîtresse des territoires qui, à la date de l'indépendance, constituaient respectivement les provinces de Honduras et du Nicaragua ». Plusieurs Etats ont ainsi consacré l'application de ce principe dans leurs constitutions nationales (SOREL et MEHDI, 1994). 
C'est ainsi que la CIJ précise dans son arrêt du 22 décembre 1986 dans l'Affaire du différend frontalier Burkina-Faso / Mali qu'il convient d'observer que le principe d'uti possidetis parait bien avoir été invoqué pour la première fois en Amérique hispanique, étant donné que c'est sur ce continent qu'on a assisté pour la première fois au phénomène de décolonisation entraînant la formation d'une pluralité d'Etats souverains sur le territoire ayant appartenu à une seule métropole. Le principe avait été mis en place dans cette partie du continent pour jouer un rôle précis.

\section{Le rôle du principe}

Ce principe avait pour rôle de protéger les nouveaux Etats indépendants des velléités impérialistes d'autres puissances coloniales, mais aussi de servir de base de délimitation de ces territoires.

Concernant le premier rôle, il était question pour les nouveaux Etats d'indiquer qu'il n'existe plus sur leur continent des espaces libres et sans maîtres, question de dissuader les éventuels conquérants. Le principe les protégeait donc d'une éventuelle appropriation de leur territoire jugé comme "'terra nullius" par certains Etats étrangers. Cela est précisé par la Cour toujours dans l'Affaire Burkina-Faso / Mali quand elle indique, en parlant du principe, que: "Sa finalité à l'époque de l'accession à l'indépendance d'anciennes colonies espagnoles d'Amérique était de priver d'effet les visées éventuelles de puissances colonisatrices non américaines sur des régions que l'ancienne métropole avait assigné à l'une ou l'autre des circonscriptions et qui étaient demeurées non occupées ou inexplorées».

Dans la même lancée, la Cour a également rappelé cela dans l'Affaire du différend frontalier terrestre, insulaire et maritime entre El Salvador et le Honduras du 11 septembre 1992 en précisant que : "Ce principe général offrait l'avantage de poser en règle absolue qu'il $n$ y'a pas en droit dans l'ancienne Amérique espagnole, de territoire sans maître et qu'il existait de nombreuses régions qui n'avaient pas été occupées par l'Espagne et de nombreuses régions inexplorées ou habitées par des indigènes non civilisés. Ces régions étaient réputées appartenir, en droit à chacune des provinces espagnoles à laquelle les territoires étaient attachés en vertu des anciennes ordonnances royales de la mère patrie espagnole. Ces territoires bien que non occupés en fait, étaient d'un commun accord considérés comme occupés en droit dès la première heure par la nouvelle République »(RSA, vol. I, p. 228).

Parlant du second rôle du principe, notamment l'utilisation du principe comme instrument de délimitation, il faut dire qu'il en a été fait bon usage. En effet, le principe avait aussi pour fonction de maintenir comme frontière internationale les anciennes limites administratives qui séparaient les circonscriptions administratives tracées par la puissance coloniale entre les nouveaux Etats indépendants. D'où Joe Verhoven pense que l'uti possidetis 
est une technique qui transforme en frontières internationales les délimitations internes qui séparent plusieurs entités lorsque celles-ci parviennent simultanément à l'indépendance, (VERHOEVEN, 2000). Ainsi, les nouveaux Etats d'Amérique latine ont fait le choix en 1810 de faire de ce principe la règle qui leur permettrait de procéder facilement à la délimitation de leur territoire. L'uti possidetis avait donc pour effet d'interdire la modification de limites tracées par les puissances coloniales, comme cela a été le cas en Afrique.

\section{B. Application du Principe de L'uti Possidetis en Afrique}

Comme indiqué ci-dessus, le principe de l'uti possidetis juris a fait ses preuves en Amérique latine lors des opérations de décolonisation. Plusieurs auteurs ont ainsi soutenu que l'uti possidetis est une règle coutumière de portée régionale, d'où COHEN Jonathan estime que «le principe de l'uti possidetis constitue peut-être une règle de droit international américain, mais en tant que principe régional, il est inopposable aux Etats tiers », (1972). "Cette très large diffusion tient principalement aux avantages préventifs et défensifs que procure en la matière le statu quo », (SOREL et MEHDI, 1994). Ainsi, en ce qui concerne les affaires africaines, c'est le principe de l'uti possidetis juris qui a été privilégié. Cela a été consacré dans l'Affaire du différend frontalier Burkina-Faso / Mali où la CIJ a estimé qu'elle ne saurait écarter le principe de l'uti possidetis dont l'application [...] a pour conséquence le respect des frontières héritées. Car ceux-ci impliquent d'une part la non remise en cause des frontières héritées de la colonisation (1) et, d'autre part, le respect de l'intégrité territoriale (2).

\section{La non remise en cause des frontières héritées de la colonisation.}

La délimitation de la frontière étant une opération juridique consistant à fixer le tracé d'une frontière entre deux Etats, elle ne peut se faire que suivant un ensemble de règles. C'est ainsi que pour déterminer les frontières terrestres des Etats africains dans les affaires qui les opposent, les juges précisent d'abord la règle de droit applicable en la matière afin que nul n'en ignore.

Les Etats africains lors de leur accession aux indépendances ont aussi décidé d'utiliser le principe de l'uti possidetis en matière de délimitation de leurs frontières (KARAMOKO OUATTARA, 2014). Ils ont entériné cela dans la résolution AGH/Res 16 (1) du 22 juillet 1964 adoptée au Caire lors de la conférence des Chefs d'Etat et de gouvernement, en indiquant que : «... tous les Etats membres s'engagent à respecter les frontières existantes au moment où ils ont accédé à l'indépendance ». Cette déclaration consiste en une interdiction faite aux membres de remettre en cause les frontières telles que laissées par les puissances coloniales. On la connait encore sous l'appellation principe de "l'intangibilité des frontières héritées de la colonisation" qui vise 
à assurer le respect des limites territoriales d'un Etat au moment de son accession à l'indépendance.

Le principe de l'intangibilité des frontières héritées de la colonisation ou uti possidetis africain, avait pour objectif d'éviter que les frontières tracées par les colons soient remises en cause, ce qui aurait été de nature à remettre en cause la stabilité et la sécurité des frontières et donc de conduire éventuellement à la rupture de la paix (ABLINE, 2006). En effet, étant donné qu'au moment des indépendances, les nouveaux Etats africains étaient confrontés aux conflits de contestation des frontières, certains dirigeants africains ont appelé à la remise en cause du tracé territorial colonial en estimant qu'il était judicieux de revenir sur les erreurs effectuées lors du découpage colonial. Les partisans de cette thèse étaient regroupés au sein du groupe dit "Groupe de Casablanca" avec pour chef de fil le Maroc. Par contre d'autres dirigeants partisans du statu quo souhaitaient le maintien du tracé hérité de la colonisation au motif que celui-ci éviterait les conflits sans fin et conduirait à la paix et la stabilité des frontières. Ces derniers étaient regroupés au sein du groupe dit "Groupe de Monrovia" avec pour chef de fil l'Afrique du Sud. C'est l'idéologie de ce dernier groupe qui a prospéré à savoir le maintien du statu quo territorial, et précisément la non remise en cause des frontières héritées de la colonisation. L'enjeu ici étant d'empêcher la survenance des conflits dus à la remise en cause des frontières.

En effet, la non remise en cause des frontières héritées de la colonisation suppose deux aspects : premièrement la non remise en cause du titre juridique sur lequel repose la frontière, ensuite la non remise en cause du tracé colonial (DEL CARMEN MARQUEZ CARRACSO, 1997). Concernant le premier aspect, la Cour Internationale de Justice est stricte à ce sujet; elle estime qu'on ne peut remettre en cause un traité colonial qui établit clairement la frontière entre deux Etats étant donné que la délimitation du territoire du nouvel Etat résulte de ce traité qu'il hérite de l'Etat prédécesseur. A cet effet, elle indique de manière péremptoire dans l'Affaire du différend territorial Jamahiriya arabe libyenne / Tchad du 3 février 1994 que : "Une frontière établie par traité acquiert ainsi une permanence que le traité lui-même ne connait pas nécessairement. Un traité peut cesser d'être en vigueur sans que la pérennité de la frontière en soit affectée». Toutefois, celle-ci tient à préciser que nonobstant ce fait, "Cela ne veut pas dire que deux Etats ne peuvent pas, d'un commun accord modifier leur frontière. Un tel résultat peut naturellement être obtenu par consentement, mais lorsqu'une frontière a fait l'objet d'un accord, sa persistance ne dépend pas de la survie du traité par lequel ladite frontière a été convenue ». De ce fait, il est assez clair qu'on ne peut remettre en cause un titre juridique (traité, accord, ordonnances, ordres royaux etc.) sur lequel repose la frontière. 
Il est également recommandé de garder le statu quo territorial en évitant de remettre en cause le tracé colonial pour maintenir la paix et la stabilité des frontières. En effet, le maintien du statu quo territorial est apparu en Afrique comme une solution de sagesse visant à préserver les acquis des peuples qui ont lutté pour leur indépendance et à éviter la rupture d'un équilibre qui ferait perdre au continent le bénéfice de tant de sacrifices. C'est donc le besoin de consolider leur indépendance qui a amené les Etats africains à consentir au respect des frontières coloniales. La non remise en cause du tracé avait donc pour objectif la stabilité et la sécurité des jeunes Etats africains. Ce qui justifie amplement l'adoption du principe de l'intangibilité des frontières héritées de la colonisation qui a pour corollaire le respect du principe de l'intégrité territoriale.

\section{Le respect de l'intégrité territoriale}

L'intégrité territoriale est un principe de droit international qui renvoie au droit et au devoir d'un Etat souverain de préserver son territoire et défendre ses frontières (CHRESTIA, 2002). Le principe de l'intégrité territoriale est le principe qui reconnaît à l'Etat le droit à une existence souveraine sur un territoire qui lui est propre et dont l'assise et les limites sont établies conformément au droit international, (EL-OUALI, 1951). Le dictionnaire de droit international indique que l'intégrité territoriale est le caractère attaché au territoire de tout Etat, qui ne doit faire l'objet d'aucune emprise tendant à le soustraire, durablement ou momentanément, à l'autorité de l'Etat (SALMON, 2000). L'intégrité du territoire est mise en exergue pour protéger le territoire des nouveaux Etats indépendants. Il a donc selon Gaël Abline un double visage, à savoir une face interne auto-immunitaire, protégeant l'Etat d'action ou d'insurrection endogènes susceptibles d'engendrer par des sécessions une atteinte ou un morcèlement de son territoire, et une face externe garantissant l'Etat contre des attaques extérieures provoquant des empiètements territoriaux pour lui assurer l'indépendance ( ABLINE, 2006).

Ces propos ont tout leur sens dans la mesure où l'intégrité du territoire est un principe qui interdit de porter atteinte au territoire de l'Etat d'une manière ou d'une autre. Il interdit de départir un Etat d'une portion de son territoire aussi bien par les mouvements sécessionnistes à l'intérieur du pays pour diviser celui-ci, que par des mouvements rebelles. L'Afrique a connu aux aurores des indépendances la montée en puissance des mouvements sécessionnistes qui s'insurgeaient contre la délimitation des frontières opérée par les colons et souhaitaient ainsi faire éclater les Etats auxquels ils appartenaient pour former leurs propres entités. Nous avons ainsi le cas de la guerre du Biafra au Nigéria qui a duré 3 ans, de 1967 à 1970 ; nous pouvons aussi noter le mouvement Sanwi en Côte-d'Ivoire qui demandait la séparation 
de la partie Sud-est de la Côte-d'Ivoire en vue de son rattachement au Ghana, il y a aussi le cas de la rébellion sécessionniste en Casamance au Sénégal.

Le principe empêche également les Etats étrangers de violer les frontières d'un autre Etat. D'où le rattachement du principe de l'intégrité territoriale à celui de l'inviolabilité de la frontière. En effet, l'inviolabilité de la frontière signifie que les frontières d'un Etat ne peuvent être franchies de force par un autre Etat en vue de porter atteinte à sa souveraineté. Un tel franchissement constitue un acte illicite au regard du droit international. L'inviolabilité des frontières préserve les Etats des éruptions des souverainetés d'autres Etats. Ce principe est donc sur le plan politique présenté comme facilitant le respect de l'intégrité territoriale des Etats (LACHAUME, 1980). Dans la mesure où quand les frontières d'un Etat ne sont pas franchies, il va de soi que l'intégrité de ce territoire est préservée. Quoique quelques fois il peut y avoir violation des frontières d'un Etat sans que l'intégrité du territoire de celui-ci soit atteinte, dans le cas où la violation est de courte durée par exemple. Tout comme il peut y avoir remise en cause de l'intégrité territoriale d'un Etat sans qu'il n'y ait eu de violation de ses frontières. Par exemple lorsque les frontières de cet Etat ont été déplacées par voie conventionnelle. Nous constatons donc que ces deux notions ne se confondent pas même si les deux poursuivent le même objectif, la préservation des frontières. C'est ainsi que l'inviolabilité apparaît comme le meilleur moyen de préserver à titre préventif l'intégrité territoriale des Etats.

Ainsi, le principe de l'intégrité territoriale tout comme celui de l'inviolabilité des frontières ont tous pour corollaire le non recours à la force dans les relations internationales, tel que consacré dans les textes internationaux, à savoir le pacte de la SDN du 28 juin 1919 dans son article 10 qui dispose que "Les membres de la société s'engagent à respecter et à maintenir contre toute agression extérieure l'intégrité territoriale et l'indépendance politique présente de tous les membres de la société », ainsi que la Charte des Nations Unies dans son article 2 paragraphe 4 qui précise que «Les membres de l'organisation s'abstiennent dans leurs relations internationales de recourir à la menace ou à l'emploi de la force, soit contre l'intégrité territoriale ou l'indépendance politique de tout Etat, soit de toute autre manière incompatible avec le but des Nations Unies ». Certains textes régionaux l'ont aussi consacré, parmi lesquels la Charte de l'Organisation de l'Unité Africaine qui exprime la volonté des Etats africains de sauvegarder et de consolider une souveraineté durement conquise ainsi que leur intégrité territoriale dans son préambule ; la Charte de Bogota de 1948 qui consacre le principe de l'intégrité territoriale dans ses articles 1 et 9 et celui de l'inviolabilité du territoire en son article 7. Ces principes sont également consacrés dans l'acte final d'Helsinki de la conférence sur la sécurité et la coopération en Europe. Un certain nombre d'organisations internationales 
l'ont tout aussi consacré, notamment l'Assemblée Générale des Nations Unies dans sa résolution 1514 du 14 décembre 1960 intitulé "Déclaration sur l'octroi et l'indépendance aux pays et peuples coloniaux"(A/RES/1514(XV)), ainsi que la résolution 2625 du 24 octobre 1970 intitulée "Déclaration relative aux principes du droit international touchant les relations amicales et la coopération entre Etats, conformément à la Charte des Nations Unies"(A /RES/2625 (XXV)).

Il est donc avéré que le respect de l'intégrité territoriale revêt une place de choix dans les relations internationales, d'où la valeur d'une norme impérative de droit international que lui accordent certains auteurs, une norme de jus cogens, (KHERAD, 2002). Ce principe est respecté dans le processus de détermination des frontières terrestres dans la mesure où il permet de protéger les frontières existantes en empêchant que ces dernières soient remises en cause.

\section{Le Titre et les Effectivites : Deux Elements Classiques de Delimitation Frontaliere Alternativement ou Concomitamment Utilises par le Juge}

Pour déterminer la frontière, le juge indique d'abord quel est le titre qui la fixe (A), tout en prenant en compte les effectivités lorsque c'est nécessaire (B), c'est dans ce sens que Paul Reuter, dans l'affaire du temple preah vihear du 15 mai 1962, indique que «Tous les conflits territoriaux, sans exception, amènent le juge à comparer et apprécier des titres et des faits d'occupation effective ».

\section{A. Le Titre Dans la Determination des Frontieres Africaines}

Lorsque les puissances coloniales délimitaient leurs zones d'influence en Afrique, elles le faisaient sur la base d'un certain nombre de textes juridiques tels que des accords de délimitation. Ces documents sont encore appelés titres juridiques. C'est ce qu'indique la CIJ dans le différend frontalier Burkina Faso/Mali quand elle précise que «la notion de titre peut également viser aussi bien tout moyen de preuve susceptible d'établir l'existence d'un droit que la source même de ce droit». Il est donc clair qu'en matière de délimitation des frontières africaines, le titre joue le rôle de source de la souveraineté (1) tout comme celui de preuve de celle-ci (2).

\section{Le titre juridique comme source de la souveraineté}

Les Etats africains ont succédé aux puissances coloniales qui, avant de quitter le continent leur ont transféré les différents instruments qui fondaient leur souveraineté sur les territoires occupés, notamment tous les titres juridiques. Par titre juridique il faut entrevoir ici tous les documents légaux qui ont permis aux puissances coloniales de s'établir en tant que souverains 
sur les territoires qu'ils occupaient. Nous avons ainsi des documents tels que des décrets royaux, lois, décrets, arrêtés et toutes les décisions émanant des autorités coloniales compétentes pour fixer l'étendue des divisions administratives (KOHEN, 2006). Ce sont donc ces différents documents encore appelés titres juridiques qui fondent la souveraineté des Etats africains sur les espaces géographiques dont ils ont hérité.

Il faut toutefois rappeler ici que, comme l'indique Marcelo Kohen, par définition, l'uti possedetis présuppose toujours l'existence d'un titre. Etant donné que c'est ce titre qu'on transfère au successeur souverain et, qui plus est, c'est lui qui assure la preuve de la souveraineté sur le territoire. Le titre est d'autant plus important qu'hormis le fait qu'il sert comme source de la souveraineté, il sert également comme preuve de la délimitation de la frontière, car c'est dans les accords de délimitation que les colons ont circonscrit leurs zones d'influence.

\section{Le titre juridique comme preuve de la souveraineté}

Le titre juridique est un document physique identifiable. Ce peut être un texte de lois, un accord, un traité, etc. Nous pouvons le voir dans le cas de l'Affaire du différend frontalier terrestre et maritime entre le Cameroun et le Nigeria, où un certain nombre de documents légaux ont été invoqués à titre de preuve, notamment:

- la convention du 29 mai 1906 concernant la délimitation de la frontière entre les possessions françaises et britanniques à l'Est du Niger dénommée Convention franco-britannique de 1906, complétée par le protocole du 19 février 1910, protocole franco-britannique de 1910 ;

- la convention du 15 mars 1894 concernant la délimitation des colonies du Congo français et du Cameroun et des sphères d'influence françaises et allemandes dans la région du lac Tchad (convention franco-allemande de 1894);

- la convention du 18 avril 1908 entre la France et l'Allemagne aux fins de confirmer le protocole du 9 avril 1908 définissant les frontières entre le Congo français et le Cameroun (convention franco-allemande de 1908) ;

- l'accord du 15 novembre 1893 concernant la frontière entre la Grande Bretagne et l'Allemagne, complété par l'accord du 19 mars 1906 concernant la frontière de Yola au Lac Tchad (accord anglo-allemand de 1906) ;

- l'accord du 11 mars 1913 entre la Grande Bretagne et l'Allemagne concernant la frontière entre le Nigeria et le Cameroun, de Yola à la mer, de même que la règlementation de la navigation sur la frontière cross (accord anglo-allemand du 11 mars 1913); 
- l'accord du 12 avril 1913 entre la Grande Bretagne et l'Allemagne relatif à la démarcation de la frontière entre Yola et la rivière Cross (accord anglo-allemand du 12 avril 1913);

- la déclaration franco-britannique du 10 juillet 1919 concernant les limites séparant les territoires sous mandat (déclaration Milner-Simon) précisée par celle du 29 novembre 1929 et du 31 janvier 1930 (déclaration Thompson-Marchand) elle-même approuvée et incorporée dans l'échange de notes du 9 janvier 1931 (HendersonFleuriau);

- l'accord de tutelle des Nations Unies pour le Cameroun britannique et le Cameroun sous administration française approuvé le 13 décembre 1946 par l'Assemblée générale ;

- l'ordre en conseil britannique du 02 août 1946, concernant l'administration du protectorat du Nigeria et du Cameroun et divisant les régions sous tutelle britannique en deux entités administratives, le Cameroun septentrional et le Cameroun méridional (ordre en conseil de 1946) ;

- la convention du 22 mai 1964 sur la création de la commission du bassin du lac Tchad (CBLT) signée par les 4 Etats riverains (Cameroun, Nigeria, Tchad, Niger) ;

- le traité de protectorat du 10 septembre 1884 entre la Grande Bretagne et les chefs et rois du vieux Calabar (traité de 1884);

- la déclaration du 04 avril 1971 (déclaration de Yaoundé II) ;

- l'accord du $1^{\mathrm{er}}$ juin 1975 (déclaration de Maroua).

Ce sont ces différents documents légaux qui ont été retenus comme titres juridiques et qui ont servi comme éléments de preuves dans l'Affaire Cameroun/Nigeria, (LABRECQUE, 2005). Cependant, le titre bénéficie d'une grande crédibilité lorsqu'il est appuyé par des effectivités.

\section{B. Les Effectivites dans le Delimitation des Frontieres Africaines}

Les effectivités dans le règlement des différends frontaliers revêtent une certaine importance. Cependant pour être retenues, elles doivent avoir un certain contenu (1) dont on peut déduire la valeur juridique (2).

\section{La constitution des effectivités}

La constitution des effectivités doit être entendue ici comme l'ensemble d'éléments qui permettent d'établir les effectivités. A cet effet, pour Marcelo Kohen les effectivités se réfèrent à des comportements qui témoignent d'une certaine maîtrise du territoire dont il est question (KOHEN, 2006). Le dictionnaire de droit international quant à lui précise qu'en matière de délimitation territoriale l'effectivité constitue un élément de preuve qu'un 
Etat peut invoquer pour démontrer son administration souveraine sur le territoire litigieux (SALMON, 2001). L'arrêt du 22 décembre 1986 dans l'Affaire du différend frontalier Burkina-Faso/Mali ne s'éloigne pas de la définition donnée par le dictionnaire quand il définit les effectivités coloniales comme étant "Le comportement des autorités administratives en tant que preuve de l'exercice effectif des compétences territoriales dans la région pendant la période coloniale ». De cette dernière définition on comprend assez clairement que les effectivités ont été réparties en deux catégories : les effectivités coloniales auxquelles se réfère l'arrêt cité provenant des actes des puissances coloniales et les effectivités postcoloniales provenant des actes des Etats successeurs (PERRIN, 2002).

Des différentes définitions évoquées, on peut dégager dans une certaine mesure des éléments constitutifs des effectivités qu'on peut répartir en deux catégories, à savoir : l'élément intentionnel et l'élément matériel. Concernant l'élément intentionnel, il marque la manifestation de la volonté d'agir sur le territoire concerné en tant que souverain. Car il y a des cas où un Etat pose des actes de souveraineté sur un territoire sans pour autant agir en tant souverain, d'où l'élément intentionnel prouve toute son importance. C'est par exemple le cas en matière de bail territorial ou de cession à bail. Concernant le bail territorial, un Etat cède au gouvernement d'un autre une portion de son territoire pour que ce dernier y exerce une activité économique sur la base d'un contrat de bail préalablement établi entre les deux. Par exemple, l'Allemagne donna à bail à l'Etat tchécoslovaque pour une période de 99 ans des espaces qui seront placés sous le modèle de zones franches et qui seront affectés au transit direct des marchandises en provenance ou à destination de cet Etat dans le port de Hambourg et de Stein.

La cession quant à elle est l'opération par laquelle un Etat confère pour un temps déterminé à un autre Etat le droit d'exercer des pouvoirs souverains sur une partie de son territoire. L'Etat cédant reste titulaire de la souveraineté et garde vocation à en récupérer la plénitude à l'issue du délai convenu. Voir par exemple le cas du gouvernement de sa majesté britannique qui céda à bail au gouvernement de la République française aux fins et conditions spécifiques dans le modèle de bail annexé au présent protocole deux terrains à choisir par le gouvernement de la République française de concert avec le gouvernement de sa majesté britannique, article 8 de la convention concernant la délimitation des colonies britanniques et françaises dans le Nord-Ouest de l'Afrique signée à Paris le 14 juin 1898.

Néanmoins, c'est l'élément matériel qui illustre le mieux la présence de l'élément intentionnel. C'est cette combinaison d'éléments qui constitue les effectivités car si l'un des éléments vient à faire défaut il serait difficile de valider les effectivités. Ainsi, concernant l'élément matériel, il s'agit d'un ensemble d'actes marquant l'exercice du pouvoir souverain. Ce sont des actes 
qui dénotent de la maîtrise ou du contrôle du territoire normalement assuré par l'exercice des fonctions étatiques à savoir les actes exécutifs, législatifs, judiciaires et administratifs (PROTIERE, 2013).

Pour illustrer l'élément matériel, l'on va s'appuyer sur la répartition établie par Marcelo Kohen dans son ouvrage 'Possession contestée et souveraineté territoriale"' (KOHEN, 1997), à savoir :

- Les actes susceptibles d'accréditer l'existence du corpus possessionis. Selon Kohen le corpus possessionis en droit international renvoie à l'exercice des fonctions étatiques dans les trois grands domaines désignés traditionnellement comme étant les fonctions administratives, exécutives, législatives et judiciaires, qui incluent :

- La présence sur le territoire

Par présence sur le territoire, on doit entrevoir deux possibilités soit la présence physique des autorités étatiques elles-mêmes sur le territoire, soit tout au moins la présence sur le territoire des insignes de l'Etat. En parlant d'insignes de l'Etat, nous faisons référence aux armoiries et au drapeau. C'est ainsi que dans l'Affaire l'île de Palmas, la seule présence du drapeau néerlandais qui flottait sur la plage et sur l'embarcation fut considérée par le juge Max Hubert comme marquant la présence de la souveraineté néerlandaise sur ce territoire.

- Le contrôle du territoire

Qui peut se manifester par la présence de l'armée et la police sur l'espace litigieux. Ces derniers procèdent à des activités telles que la surveillance des côtes, des patrouilles armées sur l'espace revendiqué.

- L'exercice des fonctions étatiques propre au souverain sur le territoire notamment :

- Les actes législatifs

Nous avons ici l'ensemble des lois, ordonnances, décrets, arrêtés et décisions prises par les autorités possesseurs. Ainsi, comme le précise Marcelo Kohen, "La législation est l'une des formes les plus frappantes de l'exercice du pouvoir souverain».

- Les actes administratifs

C'est un ensemble d'actes que les autorités administratives mettent en place pour gérer le territoire. On peut citer quelques-uns tels que : le recensement de la population, le prélèvement des impôts, le recrutement aux emplois civils et militaires, l'exécution des travaux publics, la création d'écoles et d'hôpitaux des postes de police et de douane, etc. Par exemple, Dans l'Affaire du différend frontalier Burkina Faso/Mali, la chambre a considéré que les travaux de piste constituent un élément significatif des "effectivités» susceptibles de prouver les intentions des administrateurs coloniaux. 
- Les actes judiciaires

Ce sont les décisions qui ressortent des activités des juridictions compétentes se trouvant sur le territoire litigieux, ils indiquent ainsi l'exercice par l'Etat possesseur du pouvoir judiciaire. Concernant les autorités susceptibles d'exercer le pouvoir souverain par les actes de souveraineté sur un territoire ce ne sont que les organes de l'Etat, notamment les autorités centrales et locales.

Il faut donc noter que pour que les effectivités soient prises en compte en droit international il faut un assemblage de l'élément intentionnel accompagné de l'élément matériel, précisément la volonté de l'Etat possesseur d'agir en tant que souverain en posant des actes de souveraineté sur le territoire concerné. Ce n'est que quand les effectivités sont constituées qu'elles peuvent revêtir une certaine valeur juridique.

\section{La valeur juridique des effectivités}

Les effectivités jouissent d'une grande valeur dans la délimitation du territoire, elles sont très importantes aussi bien quand le titre existe que lorsqu'il est inexistant, d'où la relation qui existe entre le titre et les effectivités.

En effet, en cas d'existence du titre, les effectivités jouent un rôle spécifique comme le rappelle la CIJ dans l'Affaire du différend frontalier Burkina-Faso / Mali en indiquant que : "Dans le cas où le fait correspondant exactement au droit, où une administration effective s'ajoute à l'uti possidetis juris, l'effectivité n'intervient en réalité que pour confirmer l'exercice du droit né d'un titre juridique. Dans le cas où le fait ne correspond pas au droit, où le territoire objet du différend est administré effectivement par un Etat autre que celui qui possède le titre juridique, il y a lieu de préférer le titulaire du titre. Dans l'éventualité où l'effectivité ne coüncide avec aucun titre juridique, elle doit inévitablement être prise en considération. Il est enfin des cas où le titre n'est pas de nature à faire apparaître de façon précise l'étendue territoriale sur lequel il porte. Les effectivités peuvent alors jouer un rôle essentiel pour indiquer comment le titre est interprété dans la pratique ». On peut déduire de cet important paragraphe de l'arrêt du 22 décembre 1986, les différents rôles que peuvent jouer les effectivités, à savoir :

La confirmation du titre

En effet, dans la première hypothèse indiquée par la Cour, les effectivités ne jouent que le rôle de constatation d'une situation juridique. Comme le mentionne Marcelo Kohen "Dans le cas d'espèce, l'effectivité n'ajoute ni ne retranche rien, elle sert uniquement à déclarer que le fait correspond au droit», (KOHEN, 1997). Dans le cas de cette hypothèse, l'effectivité appuie le titre existant, il sert d'élément supplémentaire pour attester de la crédibilité du titre. 


\section{L'interprétation du titre}

Les effectivités dans certains cas permettent de bien interpréter le titre lorsque ce dernier est obscur. D'où la Cour précise dans la quatrième hypothèse qu'il est enfin des cas où le titre juridique n'est pas de nature à faire apparaître de façon précise l'étendue territoriale sur laquelle il porte. Les effectivités peuvent alors jouer un rôle essentiel pour indiquer comment le titre est interprété dans la pratique. Dans ce cas, les effectivités servent de base à l'interprétation du titre pertinent lorsque ce dernier n'est pas assez clair.

La preuve de l'étendue du titre

Quand les limites n'ont pas été bien définies par le titre, on peut faire référence aux effectivités pour considérer l'étendue du titre. D'où Marcelo Kohen indique qu'on a plusieurs fois pris en considération l'exercice d'autorité par les organes des divisions administratives pour prouver les limites souvent mal définies de ces dernières. Toutefois, les effectivités jouent un rôle majeur en cas d'inexistence du titre (KOHEN, 1997). Dans ce cas elles constituent le titre lui-même. C'est ainsi que la Cour mentionne dans la troisième hypothèse que "Dans l'éventualité où l'effectivité ne coexiste avec aucun titre juridique, elle doit inévitablement être prise en considération». Cela a été le cas dans l'Affaire portant sur la souveraineté territoriale sur Pulau ligitan et Pulau sipadan opposant l'Indonésie à la Malaisie où il a été considéré le poids des effectivités au détriment des titres invoqués par ces parties. La Cour a noté que ces documents étaient assez vagues et donc ne pouvaient pas être considérés comme des titres véritables, c'est ainsi qu'elle a estimé qu'on se retrouvait là, selon l'arrêt de 1986, dans la troisième hypothèse ou en l'absence de titre juridique, c'est l'utilisation des effectivités qui est le critère de détermination de la souveraineté territoriale. Cela avait déjà été le cas dans l'Affaire des Minquiers et Ecrehous de 1953. Dans cette affaire, la Cour Internationale de Justice a dû trancher le différend en évaluant le poids des effectivités réalisées par chacun des Etats pour déterminer à qui revenait la souveraineté sur les îles disputées en absence d'un titre juridique pertinent.

\section{Conclusion}

L'uti possidetis ou l'intangibilité des frontières héritées de la colonisation est très tôt apparu dans le projet de paix perpétuelle qu'ont voulu garantir les dirigeants africains à l'aube des indépendances comme la règle miracle de droit applicable dans la délimitation des frontières africaines postcoloniales. Mais ce principe ne se suffisant pas à lui-même, a souvent été combiné aux titres et aux effectivités. Toutes choses qui ont construit au final le triptyque déterminant le droit de la délimitation des frontières postcoloniales. Comme nous l'avons indiqué ci-dessus, le titre est une preuve documentaire produite par les parties pour établir des droits respectifs de chacun sur le territoire querellé. Dans tous les différends frontaliers et 
territoriaux, les titres juridiques jouent un rôle fondamental, parce qu'ils sont présentés au juge pour lui servir de boussole pour procéder à la délimitation de la frontière. Car le juge ne crée pas la frontière, il l'identifie juste sur la base des documents qui l'attestent. S'agissant des effectivités il faut noter qu'elles sont importantes en matière de délimitation territoriale, dans la mesure où elles confirment le titre, c'est-à-dire lorsqu'elles coïncident avec la délimitation indiquée dans l'accord. Elles permettent une meilleure interprétation du titre quand ce dernier est obscur, et surtout, elles prouvent l'étendue du territoire à délimiter. On peut donc constater qu'en matière de délimitation des frontières africaines, les trois concepts s'imbriquent.

\section{References:}

1. ABLINE, G. (2006). Sur un nouveau principe de droit international : l'uti possedetis, Thèse de doctorat, Université d'Angers.

2. BARDONNET, D. (1976). Les frontières et les relativités de leur tracé, (problèmes choisis). Vol. 53, pp. 9-166. RCADI.

3. BASDEVANT, J. (1960). Dictionnaire de terminologie du droit international, Paris, Sirey.

4. BEDJAOUI, M. (1972). Le règlement pacifique des différends africains. Vol. 18, pp 85-99. AFDI.

5. CHRETIA, P. (2002). Le principe d'intégrité territoriale. D'un pouvoir discrétionnaire à une compétence liée. Harmattan.

6. COHEN, J.C. (1972). Les îles Falkland (Malouine). Vol.18, pp. 235262. AFDI.

7. DEL CARMEN MARQUEZ CARRACSO, M. (1997). Régime des frontières et autres régimes territoriaux face à la succession d'Etats. Dans EISENANN, P.M. et KOSKENNIEMI, M. La succession d'Etats : la codification à l'épreuve des faits. Centre d'Etude et de recherche de droit international.

8. DUPUY, P.M. (2004). Droit international public. $7^{\mathrm{e}}$ éd. Paris, précis Dalloz.

9. EL-OUALI, A. (1985). Le maintien des frontières coloniales 'l'uti possidetis"' ou le non-sens juridique du principe de base de l'OUA pour le règlement des différends territoriaux. Revue marocaine de droit et d'économie du développement.

10. KAMGA, M. (2006). Délimitation maritime sur la côte atlantique africaine, Bruylant, Bruxelles.

11. KARAMAKO OUATTARA, L. (2014). Les frontières en Afrique : héritage du passé colonial, enjeu actuel. Thinking Africa.

12. KHERAD, R. (2002). L'application de l'uti possidetis aux cas de dissolution d'Etats en Europe. Colloque de Tanger, (pp.19-35). Revue générale des collectivités territoriales. 
13. KLEIN, P. (1999). Les glissements sémantiques et fonctionnels de l'uti possedetis. Bruxelles, Bruylant.

14. KOHEN, M. (1997). Possession contesté et souveraineté territoriale. Paris, PUF.

15. KOHEN, M. (2006). Relation titre/effectivités dans le contentieux territorial à la lumière de la jurisprudence récente. $R G D I P$.

16. LABRECQUE, G. (2005). Les différends territoriaux en Afrique: règlement juridictionnel, l'Harmattan.

17. LACHAUME, J.F. (1980). Frontière et séparation, Colloque de Poitier, pp.77-94. Paris, Pedone.

18. LOULICKKI, M. (2018). L'intangibilité des frontières africaines à l'épreuve des réalités contemporaines. Policy center.

19. MEHDI, R. (1998). L'application par le juge du principe de l'uti possidetis. Dans le juge international et l'aménagement de l'espace : la spécificité du contentieux territorial. Paris, Pedone, coll. Contentieux international.

20. PERRIN, D. (2002). Titre conventionnel et effectivités : l'Affaire de la souveraineté sur le Pulau ligitan (Indonésie/Malaisie). AFDI.

21. PERSON, Y. (1972). L'Afrique noire et ses frontières. Le mois en Afrique.

22. PROTIERE, G. (2013). Espace et territoire dans la jurisprudence de la Cour internationale de Justice. Espace du droit et droit des espaces.

23. SALMON, J. (2001). Dictionnaire de droit international public. Bruylant, Bruxelles.

24. SOREL, J.M. et MEHDI, R. (1994). L'uti possidetis entre consécration juridique et la pratique : Essai de réactualisation. Vol. 40 AFDI.

25. VERHOEVEN, J. (2000). Droit international public, Bruxelles, Lacier. 